\title{
Ambient Light Organic Sensor in a Printed Complementary Organic TFT Technology on Flexible Plastic Foil
}

Giorgio Maiellaro, Egidio Ragonese, Senior Member, IEEE, Romain Gwoziecki, Stephanie Jacobs, Nenad Marjanović, Marek Chrapa, Jürg Schleuniger, and Giuseppe Palmisano, Senior Member, IEEE

\begin{abstract}
This paper presents an organic sensor for ambient light monitoring fabricated on flexible plastic foil. The sensor exploits an organic photodiode whose photocurrent is linearly converted into an output voltage by a transconductance operational amplifier in a feedback configuration. The photodiode is based on an inkjet printed bulk heterojunction blend of P3HT/PCBM sandwiched between Au and Al electrodes, whereas the amplifier is implemented in a printed complementary organic TFT technology. Both the photodiode and the amplifier were fully characterized in stand-alone configuration. Then, the response of the assembled sensor was measured by using a commercial $12-\mathrm{V}$ halogen lamp as light source. Experimental data demonstrated that the sensor is able to provide a linear detection of the incident light intensity up to 11000 lux.
\end{abstract}

Index Terms-Current-to-voltage (I/V) converter, foil bonding, organic circuits, pentacene, photodiode (OPD), thin film transistor (TFT), transconductance operational amplifier (OTA).

\section{INTRODUCTION}

$\mathbf{I}$ $\mathrm{N}$ THE LAST years, considerable research efforts have been addressed to the development of organic smart sensors for low-cost, large area or disposable applications [1]-[13] as a result of the increasing performance of organic thin-film transistor (OTFT) technology on flexible substrate. The main advantage of OTFT technology over traditional silicon manufacturing is the direct fabrication of circuits on low-cost plastic foils by using both low processing temperatures and printing methods that guarantee cost-efficient production [15], [16]. Moreover, such technologies are well suited for the fabrication on the same substrate of different kinds of organic sensors, among which temperature [1], [2], gas [3], touch/tactile sensors [4]-[8], biosensors [9]-[11], and $\mathrm{pH}$ sensors [12]-[14], often exploiting elementary cell array configurations. Although

Manuscript received June 10, 2013; revised September 03, 2013; accepted September 17, 2013. Date of publication October 30, 2013; date of current version March 25, 2014. This work was supported by the European FP7 Project FlexNet under grant agreement FlexNet 247745. This paper was recommended by Associate Editor J. M. de la Rosa.

G. Maiellaro and E. Ragonese were with DIEEI, Università di Catania, Catania, I-95125, Italy. They are now with STMicroelectronics, Catania, I-95121, Italy (e-mail: giorgio.maiellaro@st.com, egidio.ragonese@st.com).

R. Gwoziecki and S. Jacobs are with CEA-LITEN, Grenoble, F-38054, France (e-mail: romain.gwoziecki@cea.fr, stephanie.jacob@cea.fr).

N. Marjanović, M. Chrapa, and J. Schleuniger are with CSEM Muttenz, 4132, Muttenz, Switzerland (e-mail: nenad.marjanovic@csem.ch, marek.chrapa@csem.ch, juerg.schleuniger@csem.ch).

G. Palmisano is with DIEEI, Università di Catania, Catania, I-95125, Italy. (e-mail: giuseppe.palmisano@dieei.unict.it).

Digital Object Identifier 10.1109/TCSI.2013.2286031 organic semiconductor mobility is not comparable to that of poly-and mono-crystalline silicon, low speed is often compatible with most sensor applications due to the slow variation of measured physical variables. Most OTFT processes that are adopted in organic sensors still feature only p-type transistors, since very few complementary OTFT (C-OTFT) technologies are available [17]-[20].

In this scenario, organic photodiodes (OPDs) are attracting much attention of both industries and academia since they are well suited for fabrication of flexible, large-area, and low-cost devices [21]-[26]. In the last years, the integration of OPDs and TFT transistors has been already successfully exploited in array configuration to implement large-area organic imagers and scanners [27]-[29]. For such devices, on/off OPD functionality was mainly required. At the present time, state-of-the-art C-OTFT technologies allow considerably increasing both complexity and reliability of signal-conditioning interfaces of organic smart sensors [30], [31]. Under this new perspective, further applications of OPDs are becoming very promising, which are related to ambient light monitoring [23], [32]. Typical examples are light control systems for mobile device displays, in building/home environment, etc. In ambient light applications, sensors are required to detect the intensity of visible light, with performance covering the range from moonlight levels of about 1 lux to sunny daylight levels of 10.000 lux. For the sake of completeness, Table I reports some typical illuminance values under different ambient conditions.

In this paper, an organic sensor implemented on flexible foils is proposed for ambient light monitoring applications. It exploits an organic OPD and a high-gain transconductance operational amplifier (OTA) in a printed C-OTFT technology [19], [20], which guarantees a highly linear conversion of the photocurrent into the sensor output voltage. The sensor was developed as a system demonstrator within a European project on Flexible, Organic, and Large Area Electronics (FOLAE) [33].

The paper is organized as follows. Section II deals with the sensor architecture, assembling approach, design, fabrication, and stand-alone measured performance of sensor building blocks (i.e., OPD and OTA). The experimental results of the overall light sensor are discussed in Section III and main conclusions are drawn in Section IV.

\section{AMBIENT Light ORgANiC SENSOR}

The simplified block diagram of the organic light sensor is shown in Fig. 1(a). The sensor is made up of two basic building 


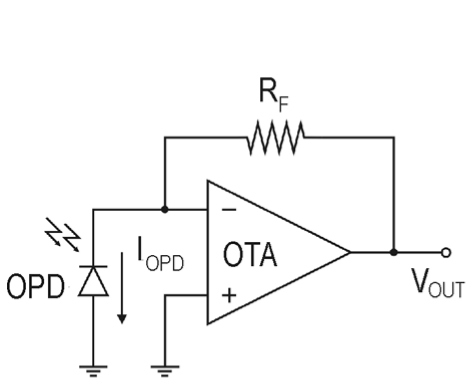

(a)

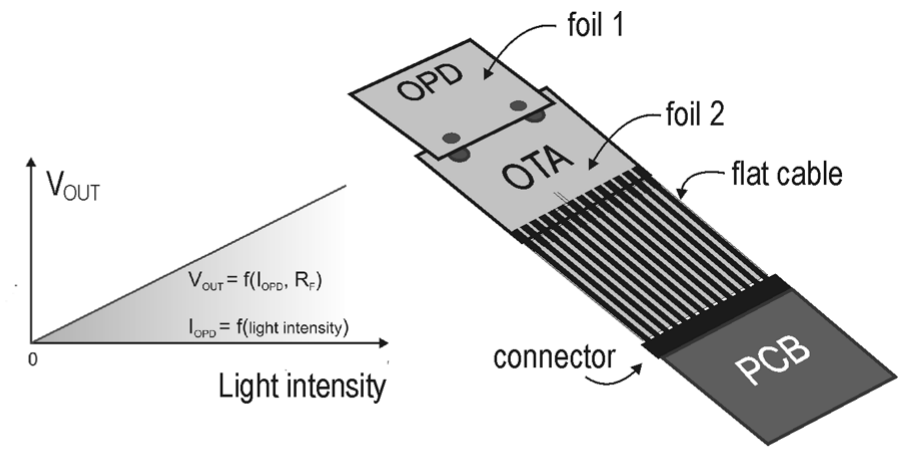

(b)

(c)

Fig. 1. (a) Simplified block diagram of the organic light sensor. (b) Linear detection of the incident light intensity. (c) Foil-to-foil assembly.

TABLE I

TyPICAL ILLUMINANCE VALUES.

\begin{tabular}{cc}
\hline Surface illuminated by & Illuminance [lux] \\
\hline Full moon on a clear night & $0.27-1.0$ \\
Twilight & 10 \\
Living room light & 50 \\
Office building hallway lighting & 80 \\
Office lighting & $320-500$ \\
Sunrise or sunset on clear day & 400 \\
Overcast day & 1000 \\
Full daylight & $10000-25000$ \\
\hline
\end{tabular}

blocks, i.e., an OPD and a transconductance operational amplifier (OTA) for signal conditioning. Specifically, the OTA is employed in a current-to-voltage (I/V) feedback configuration to convert the photodiode current, $\mathrm{I}_{\mathrm{OPD}}$, into the output voltage, $\mathrm{V}_{\text {OUT }}$. The circuit allows a linear detection of the incident light intensity to be achieved. Indeed, the operational amplifier output voltage is proportional to the OPD photocurrent, as shown in Fig. 1(b).

The light sensor implementation was achieved by using a foil-to-foil assembly, as depicted in Fig. 1(c). Both OTA and OPD were fabricated on different foils that were connected together by means of isotropic conductive glue annealed at $60^{\circ} \mathrm{C}$ for 90 minutes. Moreover, to simplify the interfacing with the lab instruments during the sensor characterization, a flexible flat cable was adopted for connecting the OTA terminals to the evaluation board (PCB). The bond between the flat cable and the foil is guaranteed by the isotropic conductive glue as well, still maintaining the flexibility of the overall system.

\section{A. Complementary Organic TFT Technology}

The adopted process is a printed complementary organic technology manufactured on an $11 \times 11 \mathrm{~cm}^{2}$ flexible foil by CEA-Liten [19], [20]. The TFTs are implemented in a top-gate bottom-contact multi-finger structure with a $20-\mu \mathrm{m}$ channel length on 125- $\mu \mathrm{m}$ thick polyethylene-naphtalate (PEN) substrate. Main electrical parameters of p-type and n-type transistors are summarized in Table II.
TABLE II

Main Electrical Parameters of C-OTFTs.

\begin{tabular}{ccc}
\hline \hline Parameters & p-type TFT & n-type TFT \\
\hline Carrier mobility $\left[\mathrm{cm}^{2} / \mathrm{V} \cdot \mathrm{s}\right]$ & 1.5 & 0.55 \\
Threshold voltage $[\mathrm{V}]$ & -20 & 18 \\
Subthreshold slope $[\mathrm{V} / \mathrm{dec}]$ & 2.4 & 1.2 \\
ION $_{\text {OFF }}$ & $\sim 10^{7}$ & $>2 \cdot 10^{7}$ \\
\hline \hline
\end{tabular}

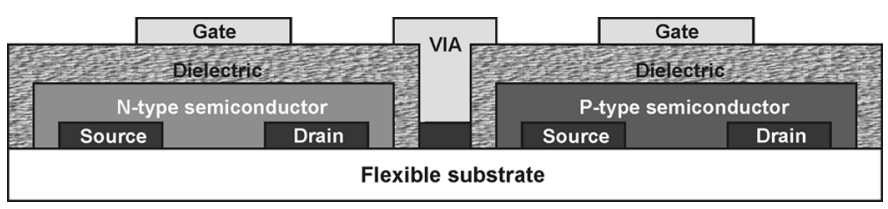

Fig. 2. C-OTFT cross-section of the adopted technology.

A simplified cross-section of both p-type and n-type TFTs is shown in Fig. 2. A photo of a complete foil along with the microphotograph of a multifinger O-TFT ( $W=2000 \mu \mathrm{m}$, $L=20 \mu \mathrm{m}$ ) is shown in Fig. 3. The C-OTFTs are based on small molecule organic semiconductors and exhibit a typical carrier mobility $\left(\mu_{\mathrm{SAT}}\right)$ of $1.5 \mathrm{~cm}^{2} / \mathrm{V} \cdot \mathrm{s}$ and $0.55 \mathrm{~cm}^{2} / \mathrm{V} \cdot \mathrm{s}$ for p-type and n-type, respectively. Since present printing resolution of metallic ink cannot reach line/spacing resolution of $20 \mu \mathrm{m}$ as well as the required flatness, a direct patterning approach by means of laser ablation was chosen to pattern the source/drain electrodes. Gold is sputtered to a thickness of $30 \mathrm{~nm}$ on the PEN and then patterned using laser beam to form the source and drain electrodes as well as the first level of interconnections. Then, the n-type organic semiconductor (Polyera ActivInk) is patterned by printing methods. The source/drain electrodes and the PEN are cleaned with an $\mathrm{O}_{2}$ plasma to prepare the surface for the self-assembled monolayer (SAM) deposition and p-type organic semiconductor (i.e., TIPS-pentacene) printing. The common fluoropolymer dielectric (CYTOP, $750 \mathrm{~nm}$ thickness) is then screen-printed on top of both semiconductors and then annealed, leaving open areas for via holes. Finally, a $5-\mu \mathrm{m}$ silver-ink conductor is screen-printed, forming the gate electrodes of OTFTs and the second level of interconnections. 


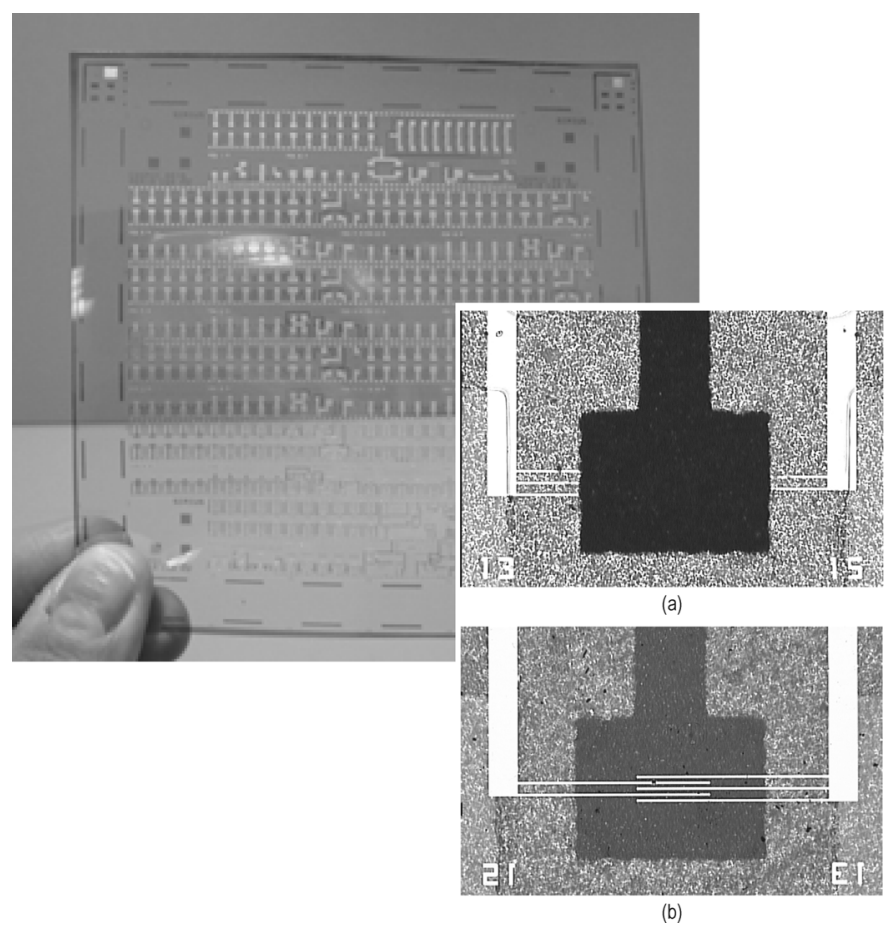

Fig. 3. Photo of a plastic foil and the details of a multifinger O-TFT. (a) Top view. (b) Bottom view ( $W=2000 \mu \mathrm{m}, L=20 \mu \mathrm{m}$ ).

\section{B. OPD on Flexible Foil}

The OPD was developed by CSEM from a previous device fabricated on glass substrate [25], [26]. A technology transfer was put in place to manufacture the OPD on the same PEN substrate adopted by the C-OTFT process, thus enabling the integration of a fully organic flexible light sensor. A redesign of the OPD was also required to adjust the photocurrent level to that delivered by the OTA (i.e., $\mu$ A range).

The OPD has an active area of $0.04 \mathrm{~cm}^{2}$. Its simplified crosssection is depicted in Fig. 4(a). The PEN substrate with semitransparent gold film $(\sim 30 \mathrm{~nm})$, acting as the OPD anode, was provided by CEA-Liten and properly patterned. A blend of bulk heterojunction P3HT:PCBM $(1: 1)$ was dissolved in a mixture of ortho-dichlorobenzene and chloroform in a concentration of $80 \mathrm{mg} / \mathrm{mL}$. A selective deposition method on top of the Au pattern was required in order to integrate the OPD with the signal conditioning circuit interface. Thus the printing was carried out with a single nozzle inkjet printer by Microdrop equipped with a MDK-140 print head, while keeping the substrate at a temperature higher than $25^{\circ} \mathrm{C}$. The diameter of the nozzle was $50 \mu \mathrm{m}$. The printing was performed in ambient conditions. To get a fairly thick film of approximately $800 \mathrm{~nm}$, the printing resolution was set to 300 dots per inch (DPI). This thick film was used for minimizing short-cuts, decreasing the off current, and increasing the on/off ratio. After printing, annealing on a hotplate in a $\mathrm{N}_{2}$-glovebox was done. The 100 -nm Al cathode was thermally evaporated through shadow mask by thermal vacuum evaporation. The fabricated devices were tested using Keithley 2400 in dark and under illumination in $\mathrm{N}_{2}$-glovebox.

Finally, the OPDs were encapsulated using a flexible semitransparent pouch technology, as depicted in Fig. 4(b). It consists of a transparent flexible barrier film $\left(0.02 \mathrm{~g} /\left[\mathrm{m}^{2} / 24 \mathrm{~h}\right]\right.$ at $38^{\circ} \mathrm{C} 100 \% \mathrm{rH}$ ) on the top side, and of aluminum laminate with integrated moisture absorber on the bottom side. In order to improve encapsulation, an additional moisture getter was inserted into the OPD package. A photo of the fabricated OPD on PEN foil is shown in Fig. 4(c).

Typical $I-V$ characteristics of the fabricated OPDs in air under different illumination conditions are shown in Fig. 5. Several samples were fabricated, which allowed a high repeatability of the OPD behaviour to be experimentally verified. For this testing, a commercial halogen lamp (i.e., PHILIPS Brilliant Line $50 \mathrm{~W}, 12 \mathrm{~V}, 36$ degree) was used as light source. The distance between the lamp and the OPD was $45 \mathrm{~cm}$. The incident light intensity was varied and measured using a high precision lux meter (i.e., ISO-TECH ILM 1335).

\section{Design and Measured Performance of the C-OTFT OTA}

A classical 2-stage OTA was adopted for the implementation of the light sensor, whose performance in the C-OTFT technology was previously demonstrated [30]. Fig. 6 depicts the amplifier schematic along with transistor sizing and photo of the fabricated sample before OPD foil bonding.

$\mathrm{N}$-type input differential pair (M1-M2) was used for a lower input offset voltage, since the threshold voltage dispersion of n-type transistors is lower than the p-type ones in the adopted technology [20]. Moreover, the p-type common-source output gain stage (M5) can better provide the OPD current ( $\mathrm{I}_{\mathrm{OPD}}$ ) flowing through the feedback resistor $\left(\mathrm{R}_{\mathrm{F}}\right)$, thanks its higher mobility. The maximum output current provided by the operational amplifier can be set by properly sizing M5. To minimize the systematic input offset, the current density in M3-M5 was set according to the well-know pseudo-mirror rule, i.e., $\mathrm{I}_{\mathrm{D} 4} /[W / L]_{4}=\mathrm{I}_{\mathrm{D} 5} /[W / L]_{5}$. The amplifier design was carried out by taking advantage of a dedicated transistor model [34], which was enriched with parasitic capacitances due to layout interconnections, to well estimate the frequency behavior.

The OTA layout exploits only two metal layers for connections. It was arranged to improve transistor matching for the differential pair and current mirrors, while reducing the parasitic capacitances on critical circuit nodes responsible for non-dominant poles. The amplifier area is $15 \mathrm{~mm} \times 20 \mathrm{~mm}$ including the pads used for the OPD connection.

The amplifier was designed by considering the I/V feedback configuration and both the OPD and output load effects that are shown in the simplified schematic in Fig. 7. Specifically, an output load capacitance, $\mathrm{C}_{\mathrm{L}}$, of $30 \mathrm{pF}$ was estimated, mainly due to the parasitic capacitance of the flexible flat cable and the external components (i.e., PCB and output buffer) used for characterization. Moreover, an equivalent circuit for the organic OPD was considered, which consists of a current generator, $\mathrm{I}_{\mathrm{OPD}}$, and a shunt capacitance, $\mathrm{C}_{\mathrm{OPD}}$. At maximum illumination condition (i.e., around 11500 lux), the OPD typically draws a maximum current between 1 to $3 \mu \mathrm{A}$, while exhibiting an overall capacitance from 0.5 to $1.5 \mathrm{nF}$ with a reverse bias voltage around $2 \mathrm{~V}$. The feedback resistor, $R_{F}$, was sized as high as $680 \mathrm{k} \Omega$ in order to achieve a maximum output voltage variation $\left(\Delta \mathrm{V}_{\text {OUT }}\right)$ from 0.7 to $2 \mathrm{~V}$ for an $\mathrm{I}_{\mathrm{OPD}}$ maximum current from 1 to 3 $\mu \mathrm{A}$. Due to both $\mathrm{R}_{\mathrm{F}}$ and the large OPD capacitance, the OTA needs to be properly compensated to guarantee a stable behavior 


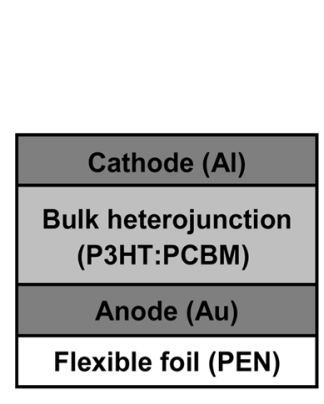

(a)

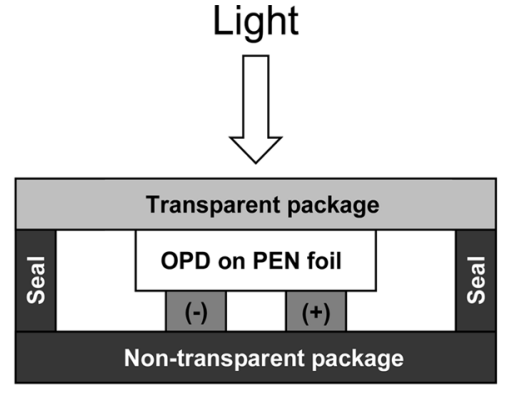

(b)

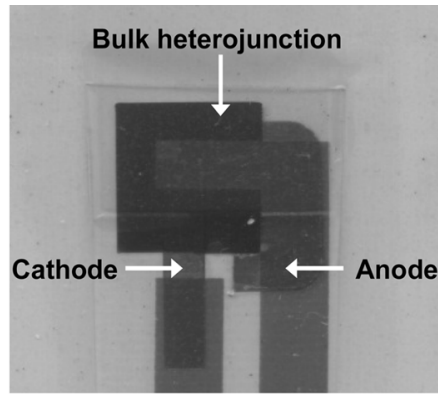

(c)

Fig. 4. (a) Simplified cross-section of the OPD. (b) Encapsulation of the OPD. (c) Photo of the fabricated OPD.

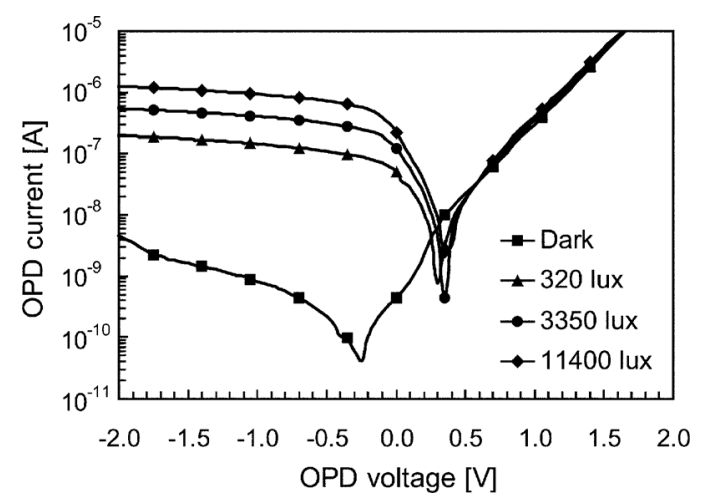

Fig. 5. OPD $I-V$ characteristics in air under various illumination intensities.

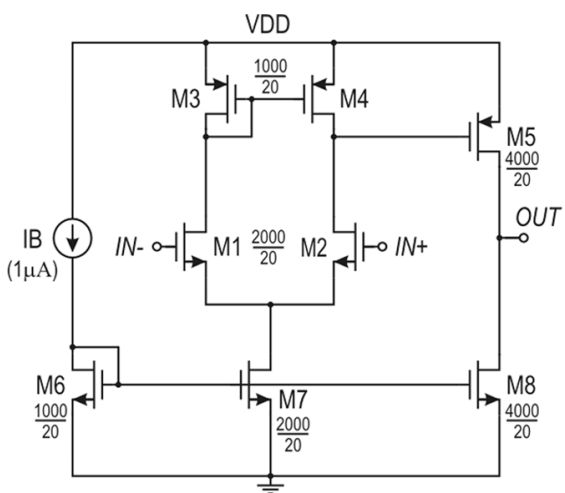

(a)

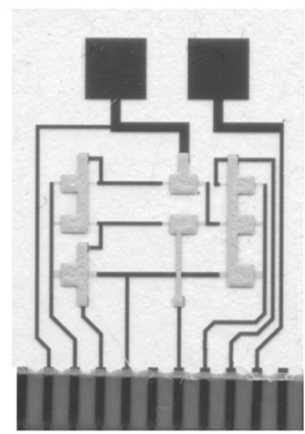

(b)
Fig. 6. 2-stage OTA for the light sensor. (a) Circuit schematic. (b) Photo of fabricated OTA before OPD foil bonding.

for the light sensor. To this aim, Miller compensation with a $\mathrm{C}_{\mathrm{C}}-\mathrm{R}_{\mathrm{C}}$ series network (i.e., $400 \mathrm{pF}$ and $2 \mathrm{M} \Omega$, respectively) was placed between the gate of M5 and the output. At a nominal supply voltage, VDD, of $60 \mathrm{~V}$, the simulated dc gain, gain-bandwidth-product (GBW), and phase margin (PM) of the whole light sensor were $60 \mathrm{~dB}, 57 \mathrm{~Hz}$, and $60^{\circ}$, respectively.

The stand-alone OTA was tested using a dedicated PCB with the required passive components, which were connected by means of a flexible flat cable, thus allowing a straightforward interfacing between amplifier and instrumentations.

Experimental characterization was performed in air at ambient temperature/pressure conditions. Measurements of the dc input-output characteristic and output short-circuit current were carried out in open-loop configuration at the three supply voltages, $40 \mathrm{~V}, 50 \mathrm{~V}$, and $60 \mathrm{~V}$, and with the non-inverting input at

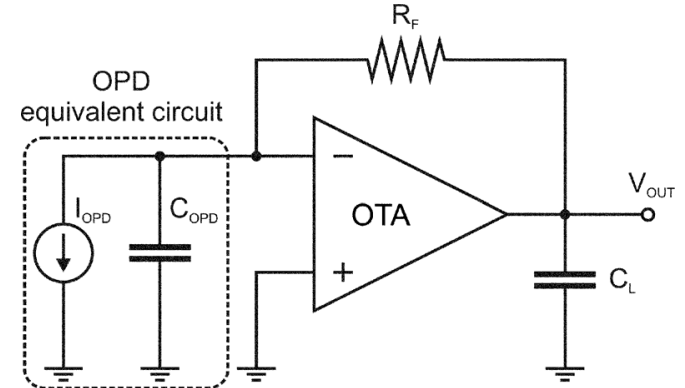

Fig. 7. Schematic of the light sensor with an equivalent circuit for the OPD.

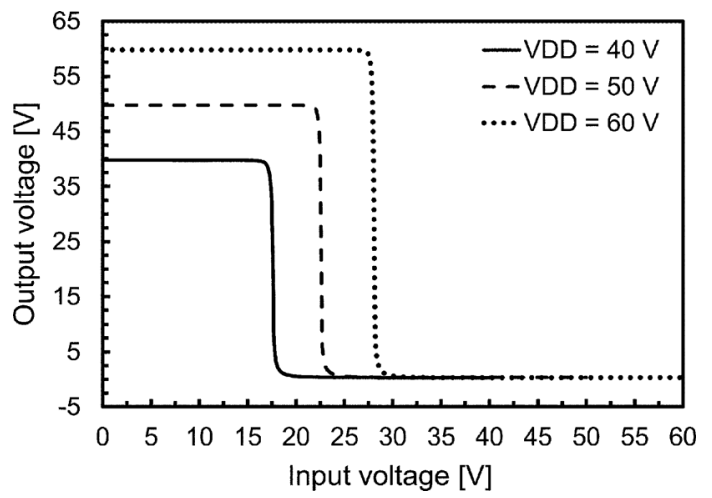

Fig. 8. Measured output voltage versus input voltage for the OTA in open-loop configuration.

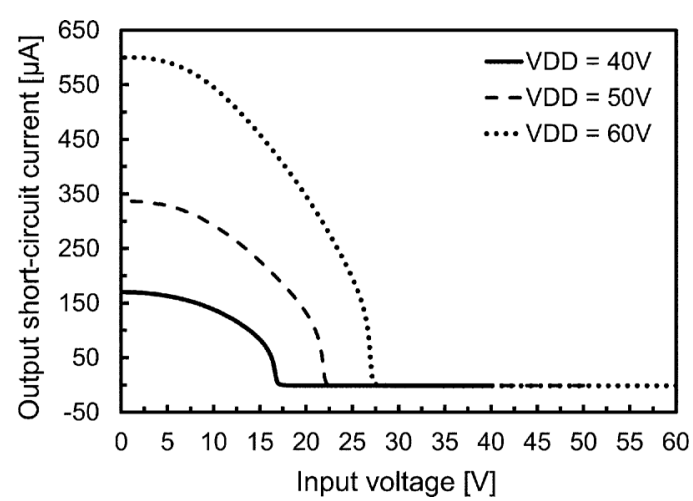

Fig. 9. Measured output short-circuit current versus input voltage for the OTA in open-loop configuration.

the bias voltages, $23 \mathrm{~V}, 28 \mathrm{~V}$, and $33 \mathrm{~V}$, respectively. In these conditions, the inverting input was swept from 0 to VDD. The measured curves for output voltage and short-circuit current are shown in Figs. 8 and 9, respectively. 


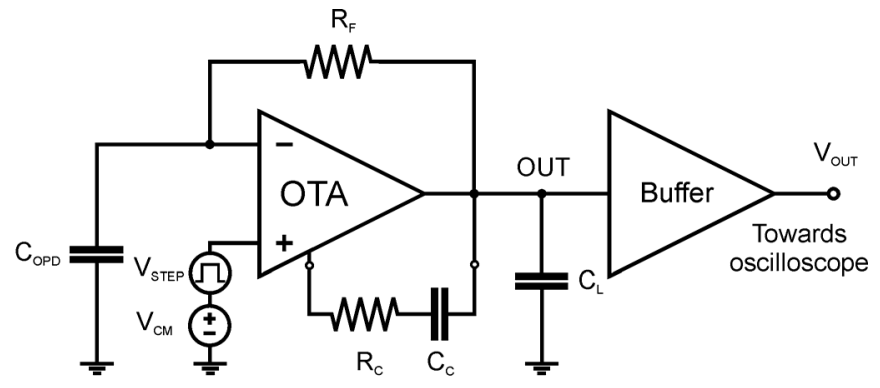

(a)

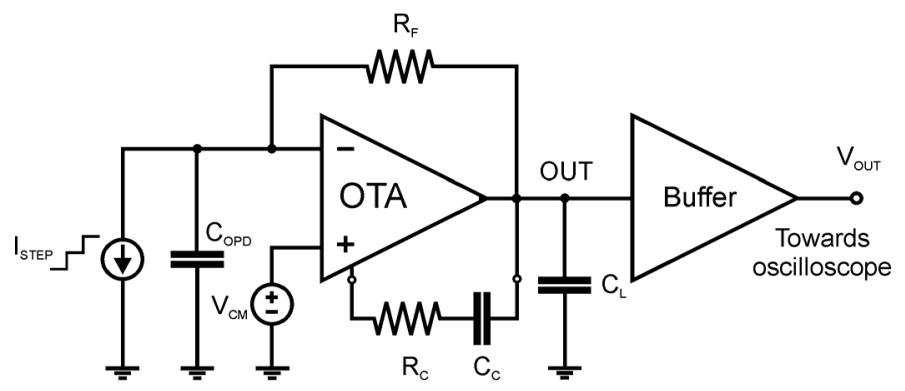

(b)

Fig. 10. Measurement set-up of the OTA in closed-loop configuration. (a) Voltage-step response. (b) Current-step response.

The dc open-loop gain achieves $47 \mathrm{~dB}, 51 \mathrm{~dB}$, and $54 \mathrm{~dB}$ at supply voltage of $40 \mathrm{~V}, 50 \mathrm{~V}$, and $60 \mathrm{~V}$, respectively. As expected, the gain is high enough to guarantee accuracy to the light sensor. At the nominal power supply of $60 \mathrm{~V}$, the measured current consumption was $6.3 \mu \mathrm{A}$ with bias current IB of $1 \mu \mathrm{A}$ and the input offset voltage was around $5.6 \mathrm{~V}$ in the measured sample. The offset is mainly due to the TFT threshold voltage mismatch that was experimentally analyzed for our C-OTFT technology in [20] and [35].

The bias current of M8 limits the output sinking current of the amplifier to $3.5 \mu \mathrm{A}$. This current was set along with the aspect ratio of transistor M5 to guarantee a nominal PM value of $60^{\circ}$. Instead, the source output current is much higher than that required by the OPD. This current depends on both the aspect ratio of M5 and its maximum gate-source voltage that in turn depends on the power supply. By setting the supply voltages at $40 \mathrm{~V}, 50 \mathrm{~V}$, and $60 \mathrm{~V}$, an output source current of $170 \mu \mathrm{A}$, $337 \mu \mathrm{A}$, and $600 \mu \mathrm{A}$, was measured. The OTA performance was also experimentally verified in light sensor configuration by including in the PCB the feedback resistor $\mathrm{R}_{\mathrm{F}}(680 \mathrm{k} \Omega)$, the equivalent OPD capacitance $\mathrm{C}_{\mathrm{OPD}}(1.5 \mathrm{nF})$, and the nominal Miller compensation network (i.e., $\mathrm{R}_{\mathrm{C}}=2 \mathrm{M} \Omega$ and $\mathrm{C}_{\mathrm{C}}=400 \mathrm{pF}$ ). The amplifier was measured at nominal biasing conditions (i.e., $\mathrm{VDD}=60 \mathrm{~V}, \mathrm{IB}=1 \mu \mathrm{A}, \mathrm{V}_{\mathrm{CM}}=33 \mathrm{~V}$ ) by using the experimental setup configurations shown in Fig. 10. The output load parasitic capacitance, $\mathrm{C}_{\mathrm{L}}$, due to the flat cable, $\mathrm{PCB}$, and output buffer was measured to be slightly higher than estimated (i.e., $39 \mathrm{pF}$ instead of $30 \mathrm{pF}$ ). A high voltage output buffer (i.e., Texas Instrument OPA-445) was used to avoid the detrimental loading effect of the oscilloscope input impedance. According to the schematic in Fig. 10(a), a square-wave signal, $V_{\text {STEP }}$, with peak-to-peak amplitude of $1 \mathrm{~V}$ and frequency of $5 \mathrm{~Hz}$ was fed into the non-inverting input to evaluate the amplifier step-response that is shown in Fig. 11. Measurements confirmed

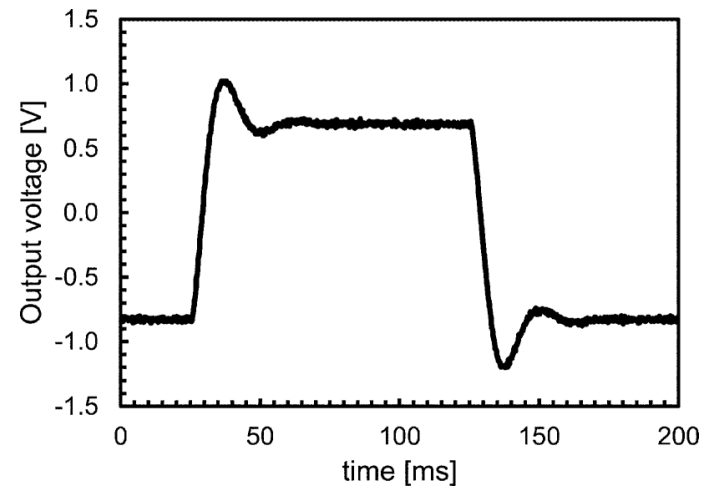

Fig. 11. Measured output voltage step-response for the OTA in closed-loop configuration.

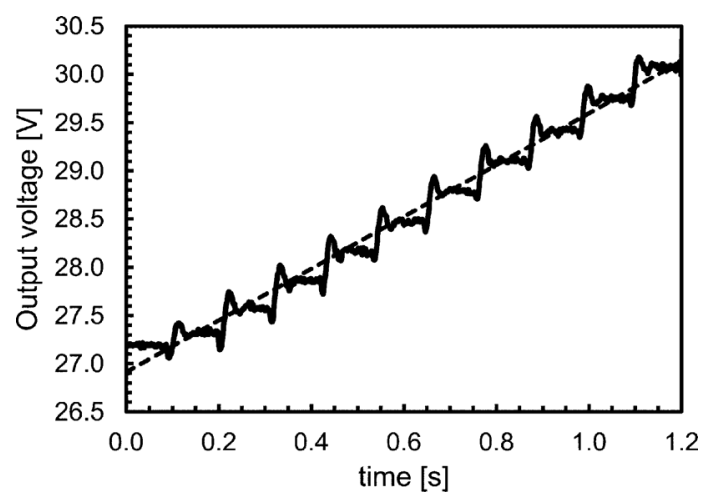

Fig. 12. Measured output voltage for the OTA in closed-loop configuration by applying an input staircase current.

closed-loop stability and a settling time around $60 \mathrm{~ms}$, which is mainly imposed by GBW and PM. However, the slow time response is not critical for the specific application since the incident ambient light intensity is a slowly variable signal. OTA time-domain measurement was also performed by applying a staircase current at the inverting input (i.e., ten steps from $0 \mu \mathrm{A}$ to $5 \mu \mathrm{A}$ with a step size of $0.5 \mu \mathrm{A}$ ), as shown in Fig. 10(b). The measured output voltage variation is shown in Fig. 12. The amplifier works as a linear $\mathrm{I}-\mathrm{V}$ converter and provides an output voltage variation that is proportional to the input current. Stability and accuracy are also confirmed.

\section{Ambient Light Organic Sensor Measurements}

The light sensor was implemented according to the foil-tofoil assembling approach sketched in Fig. 1(c). Top and bottom views of the complete sensor are shown in Fig. 13. Isotropic conductive paste was used to glue both OPD and OTA and also the light sensor to the flexible flat cable. As already done for the stand-alone OTA measurements, a dedicated PCB was used for the external passive components (i.e., feedback resistance $\mathrm{R}_{\mathrm{F}}$ and compensation network $\mathrm{R}_{\mathrm{C}}, \mathrm{C}_{\mathrm{C}}$ ) of the light sensor and for the interface circuitry, which connects the sensor with the lab instrumentations. It is worth noting that on-board passive components (i.e., $R_{F}, R_{C}$ and $C_{C}$ ) could be integrated along with TFTs on the same PEN substrate. Indeed, capacitors are naturally included in the adopted C-OTFT process, while optional carbon-ink resistors can be added to the basic process flow. Moreover, feasibility of a complete integration of OPD, active circuitry, and passive components on the same PEN foil was also 

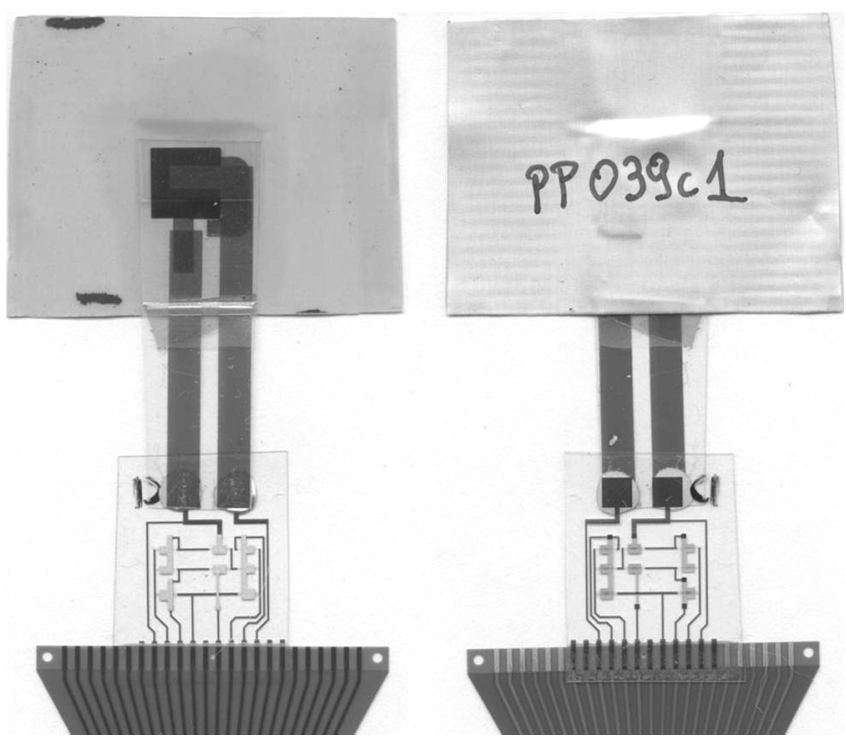

Fig. 13. Top and bottom photos of the light sensor.

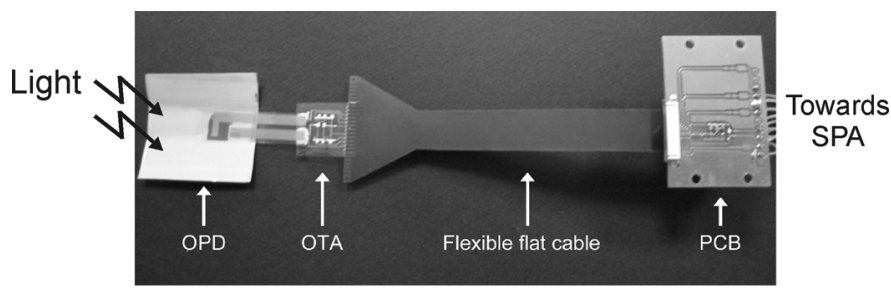

(a)

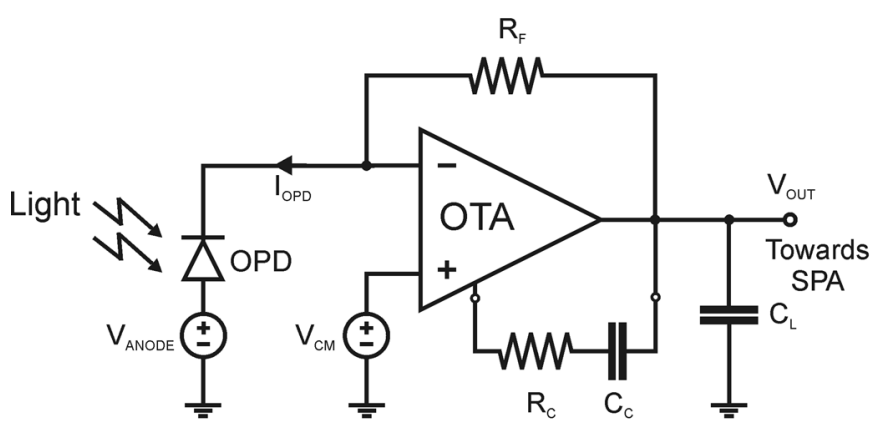

(b)

Fig. 14. Measurement setup used for the light sensor characterization. (a) photo, (b) block diagram.

verified without experiencing any performance degradation of photodiode and TFTs. However, as a first step towards a fully integrated light sensor on a single foil, the use of on-board passive components was preferred. Indeed, the absolute accuracy of integrated passive components is not adequate for the design of a compensation network that has to guarantee closed-loop stability.

The photograph of the adopted measurement set-up and the corresponding block diagram are shown in Fig. 14(a) and (b), respectively. The experimental characterization of the overall foil-to-foil light sensor was performed in air at ambient temperature/pressure conditions inside an $80 \mathrm{~cm} \times 60 \mathrm{~cm} \times 60 \mathrm{~cm}$ black box, using the same set-up adopted for the OPD measurements, i.e., the PHILIPS lamp placed at $45 \mathrm{~cm}$ to the sensor. The incident light intensity was measured using a high precision

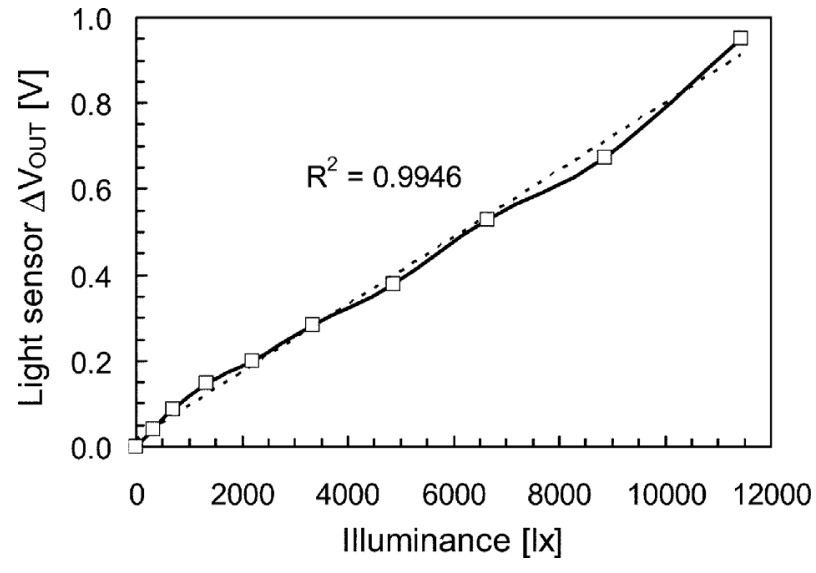

Fig. 15. Measured output voltage of the foil-to-foil light sensor at ten values of the incident light intensity ( $\mathrm{R}^{2}$ is the coefficient of determination).

lux meter (i.e., ISO-TECH ILM 1335). It was swept from 0 to $11400 \mathrm{~lx}$, thus covering the typical range required for ambient light monitoring applications. A semiconductor parameter analyzer (SPA) was used to properly set and measure all required voltages and currents, including the light sensor output voltage, VOUT.

The supply voltage, the bias current, and the common-mode bias voltage were set at $60 \mathrm{~V}, 1 \mu \mathrm{A}$, and $33 \mathrm{~V}$, respectively. The measured current consumption was $7.5 \mu \mathrm{A}$. Nominal values for the feedback resistor (i.e., $680 \mathrm{k} \Omega$ ) and the Miller compensation network ( $2 \mathrm{M} \Omega$ and $400 \mathrm{pF}$ ) were adopted. For a proper operation as light detector, the OPD was biased in reverse condition at a bias voltage, $\mathrm{V}_{\mathrm{OPD}}$, of about $-2 \mathrm{~V}$. The OTA input offset, $\mathrm{V}_{\mathrm{OS}}$, was measured and taken into account to properly set the anode voltage as follows $\mathrm{V}_{\mathrm{ANODE}}=\left(\mathrm{V}_{\mathrm{CM}}+\mathrm{V}_{\mathrm{OS}}\right)-\left|\mathrm{V}_{\mathrm{OPD}}\right|$.

The light sensor functionality was verified by measuring the output voltage variation $\left(\Delta \mathrm{V}_{\text {OUT }}\right)$ versus the incident light intensity variation. Fig. 15 depicts the light sensor output voltage $\Delta \mathrm{V}_{\text {OUT }}$ measured at ten values of the light intensity from 0 (dark condition) to $11400 \mathrm{~lx}$ (maximum illumination conditions). As expected, a linear detection of the light intensity was achieved. Indeed, an output voltage variation of about $1 \mathrm{~V}$ was measured with a photocurrent in dark and maximum illumination conditions of $20 \mathrm{nA}$ and $1.45 \mu \mathrm{A}$, respectively. For the sake of completeness, the light sensor functionality was also verified at lower power supply voltages (i.e., $40 \mathrm{~V}$ and $50 \mathrm{~V}$ ) achieving similar behaviors.

To the author' knowledge this is the first air stable ambient light organic sensor fabricated using a complementary organic TFT technology on flexible foil.

\section{CONCLUSION}

For the first time, a light sensor has been implemented using fully organic technology on flexible substrate. The sensor takes advantage of a customized OPD whose photocurrent is converted into an output voltage by a high-gain C-OTFT OTA in $\mathrm{I} / \mathrm{V}$ feedback configuration. By considering typical range of ambient light monitoring applications, the sensor provides linear detection of the incident light intensity, as experimentally demonstrated. Thanks to the adopted foil-bonding technique, the flexibility of the overall systems is also preserved. 
The work demonstrates the improved maturity of organic electronics, which is now able to deal with a complete electronic chain (i.e., from system design and component fabrication, till system assembly and characterization), thus finally behaving as conventional silicon electronics.

\section{ACKNOWLEDGMENT}

The authors would like to thank F. Carrara for his valuable suggestions in the sensor conceive phase. A special thanks is also given to A. Castorina, STMicroelectronics, Catania, Italy for his precious assistance in carrying out measurements.

\section{REFERENCES}

[1] M. Zirkl, G. Scheipl, B. Stadlober, A. Haase, G. Jakopic, J. R. Krenn, A. Sawatdee, P. Bodo, and P. Andersson, "Fully printable, flexible, large area organic optothermal sensors for human-machine-interfaces," IEEE Sensors J., pp. 1628-1631, Oct. 2009.

[2] D. He, I. Nausieda, K. Ryu, A. Akinwande, V. Bulovic, and C. Sodini, "An integrated organic circuit array for flexible large-area temperature sensing," in Proc. IEEE Int. Solid-State Circuits Conf. Tech. Dig., Feb. 2010, pp. 142-143.

[3] H. Zan, W. Tsai, Y. Lo, Y. Wu, and Y. Yang, "Pentacene-based organic thin film transistors for ammonia sensing," IEEE Sensors J., vol. 12, no. 3, pp. 594-601, Mar. 2012.

[4] T. Someya, Y. Kato, T. Sekitani, S. Iba, Y. Noguchi, Y. Murase, H. Kawaguchi, and T. Sakurai, "Conformable, flexible, large-area networks of pressure and thermal sensors with organic transistor active matrixes," Proc. Natl. Acad. Sci., vol. 102, no. 35, pp. 12321-12325, 2005.

[5] H. Kawaguchi, T. Someya, T. Sekitani, and T. Sakurai, "Cut-and-paste customization of organic FET integrated circuit and its application to electronic artificial skin," IEEE J. Solid-State Circuits, vol. 40, pp. 177-185, 2005.

[6] T. Someya, "Ambient electronics with organic transistors," in Proc. IEEE Symp. VLSI Circuits, Jun. 2007, pp. 116-119.

[7] P. Cosseddu, L. Basiricò, A. Loi, S. Lai1, P. Maiolino, E. Baglini, S. Denei, F. Mastrogiovanni, G. Cannata, and A. Bonfiglio, "Inkjet printed organic thin film transistors based tactile transducers for artificial robotic skin," in Proc. IEEE RAS/EMBS Int. Conf. Biomedical Robotics Biomechatron., Jun. 2012, pp. 1907-1912.

[8] H. Marien, M. S. J. Steyaert, E. van Veenendaal, and P. Heremans, "Analog building blocks for organic smart sensor systems in organic thin-film transistor technology on flexible plastic foil," IEEE J. SolidState Circuits, vol. 47, no. 7, pp. 1712-1720, Jul. 2012.

[9] R. P. Singh and J.-W. Choi, "Biosensors developments based on potential target of conducting polymers," Sens. Transducers J., vol. 104, no. 5, pp. 1-18, May 2009.

[10] G. Scarpa, A.-L. Idzko, A. Yadav, E. Martin, and S. Thalhammer, "Toward cheap disposable sensing devices for biological assays," IEEE Trans. Nanotechnol., vol. 9, no. 5, pp. 527-532, Sep. 2010.

[11] F. Yan, S. M. Mok, J. Yu, H. L. W. Chan, and M. Yang, "Label-free DNA sensor based on organic thin film transistors," Biosens. Bioelectron., vol. 24, pp. 1241-1245, 2009.

[12] C. Bartic, B. Palan, A. Campitelli, and G. Borghs, "Monitoring pH with organic-based field-effect transistors," Sensor Actuat. B-Chem., vol. 83 , pp. $115-122,2002$.

[13] A. Loi, I. Manunza, and A. Bonfiglio, "Flexible, organic, ion-sensitive field-effect transistor," Appl. Phys. Lett., vol. 86, no. 10, pp. 103512-103513, 2005

[14] T. Ji, P. Rai, S. Jung, and V. K. Varadan, "In-vitro evaluation of flexible $\mathrm{pH}$ and potassium ion-sensitive organic field effect transistor sensors," Appl. Phys. Lett., vol. 92, pp. 233304-233306, 2008.

[15] R. Parashkov, E. Becker, T. Riedl, H.-H. Johannes, and W. Kowalsky, "Large area electronics using printing methods," Proc. IEEE, vol. 93, no. 7, pp. 1321-1329, Jul. 2005.

[16] J. Chang, G. Tong, and E. Sanchez-Sinencio, "Challenges of printed electronics on flexible substrates," in Proc. IEEE MWSCAS, Aug. 2012, pp. $582-585$.

[17] H. Klauk, M. Halik, U. Zschieschang, F. Eder, D. Rohde, G. Schmid, and C. Dehm, "Flexible organic complementary circuits," IEEE Trans. Electron Devices, vol. 52, no. 4, pp. 865-873, Mar. 2011.

[18] S. De Vusser, S. Steudel, K. Myny, J. Genoe, and P. Heremans, "A 2 V organic complementary inverter," in Proc. IEEE Int. Solid-State Circuits Conf. Tech. Dig., Apr. 2005, pp. 618-622.
[19] A. Daami, C. Bory, M. Benwadih, S. Jacob, R. Gwoziecki, I. Chartier, R. Coppard, C. Serbutoviez, L. Maddiona, E. Fontana, and A. Scuderi, "Fully printed organic CMOS technology on plastic substrates for digital and analog applications," in Proc. IEEE Int. Solid-State Circuits Conf. Tech. Dig., Feb. 2011, pp. 328-330.

[20] S. Jacob et al., "High performance printed N and P-type OTFTs for complementary circuits on plastic substrate," in Proc. IEEE Eur. SolidState Device Res. Conf., Sep. 2012, pp. 173-176.

[21] E. S. Zaus, S. Tedde, T. Rauch, J. Fürst, and G. H. Döhler, "Design of highly transparent organic photodiodes," IEEE Trans. Electron Devices, vol. 55, no. 2, pp. 681-684, Feb. 2008

[22] Y. Ohmori and H. Kajii, "Organic devices for integrated photonics," Proc. IEEE, vol. 97, no. 9, pp. 1627-1536, Sep. 2009.

[23] J. W. Jeong, Y. W. Park, T. H. Park, J. H. Choi, H. J. Choi, E. H. Song, J. I. Lee, H. Y. Chu, and B. K. Ju, "The emission properties of integrated organic light emitting diodes with organic photo sensor for emotional lighting applications," IEEE Trans. Electron Devices, vol. 32, no. 3, pp. 348-350, Mar. 2011.

[24] J. Verilhac, "Recent developments of solution-processed organic photodetectors," in Proc. IEEE ISCDG, Sep. 2012, pp. 101-106.

[25] L. Bürgi, R. Pfeiffer, M. Mücklich, P. Metzler, M. Kiy, and C. Winnewisser, "Optical proximity and touch sensors based on monolithically integrated polymer photodiodes and polymer LEDs," Elsevier Organic Electronics, vol. 7, no. 2, pp. 114-120, Apr. 2006.

[26] M. Ramuz, L. Bürgi, C. Winnewisser, and P. Seitz, "High sensitivity organic photodiodes with low dark currents and increased lifetimes," Elsevier Organic Electronics, vol. 9, pp. 369-376, Jun. 2008.

[27] T. Someya, Y. Kato, S. Iba, Y. Noguchi, T. Sekitani, H. Kawaguchi, and T. Sakurai, "Integration of organic FETs with organic photodiodes for a large area, flexible, and lightweight sheet image scanners," IEEE Trans. Electron Devices, vol. 52, no. 11, pp. 2502-2511, Nov. 2005.

[28] I. Nausieda, K. Ryu, I. Kymissis, A. I. Akinwande1, V. Bulovic, and C. G. Sodini, "An organic imager for flexible large area electronics," in Proc. IEEE Int. Solid-State Circuits Conf. Tech. Dig., Feb. 2007, pp. $72-73$.

[29] S. Aihara, H. Seo, M. Namba, T. Watabe, H. Ohtake, M. Kubota, N. Egami, T. Hiramatsu, T. Matsuda, M. Furuta, H. Nitta, and T. Hirao, "Stacked image sensor with green-and-red-sensitive organic photoconductive films applying zinc oxide thin-film transistors to a signal readout circuit," IEEE Trans. Electron Devices, vol. 56, no. 11, pp. 2570-2576, Nov. 2009

[30] G. Maiellaro, E. Ragonese, A. Castorina, S. Jacob, M. Benwadih, R. Coppard, E. Cantatore, and G. Palmisano, "High-gain operational transconductance amplifiers in a printed complementary organic TFT technology on flexible foil," IEEE Trans. Circuits and Syst.-I, 2013.

[31] S. Abdinia, M. Benwadih, R. Coppard, S. Jacob, G. Maiellaro, G. Palmisano, M. Rizzo, A. Scuderi, F. Tramontana, A. van Roermund, and E. Cantatore, "A 4 b ADC manufactured in a fully-printed organic complementary technology including resistors," in Proc. IEEE Int Solid-State Circuits Conf. Tech. Dig., Feb. 2013, pp. 106-107.

[32] C.-T. Chiang, "Design of CMOS monolithic digitized light transducer with calibration technique for ambient light sensor applications," IEEE Sensors J., vol. 13, no. 5, pp. 1931-1940, May 2013.

[33] Flexnet Website, [Online]. Available: http://www.noe-flexnet.eu

[34] F. Torricelli et al., "Physically-based compact model of staggered $p$ and n-type organic thin film transistors," in Proc. Int. Thin-Film Transistor Conf., 2012, pp. 116-116, Org11.

[35] S. Jacob et al., "High performance printed $\mathrm{N}$ and P-type OTFTs enabling digital and analog complementary circuits on flexible plastic substrate,” Elsevier Solid-State Electron., pp. 167-178, Jun. 2013.

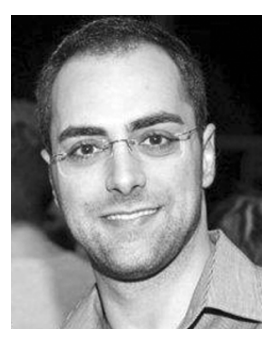

Giorgio Maiellaro received the Laurea degree in electronic engineering and the Ph.D. degree in electronics, automation, and control of complex systems engineering from the University of Catania, Italy, in 2007 and 2013, respectively.

From 2007 to 2011, he was with the Radio Frequency Advanced Design Center (RF-ADC), a joint research center supported by University of Catania and STMicroelectronics, where he was involved in the design and characterization of analog and digital circuits based on organic semiconductor as well as in the design and characterization of battery-less RF front-end for wireless sensor network in 130-nm CMOS technology. In December 2011, he joined STMicroelectronics, Catania, Italy, where he is currently involved in the design and development of $24 / 77-\mathrm{GHz}$ ICs for automotive radar sensor applications. 


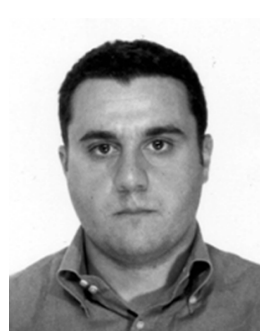

Egidio Ragonese (M'03-SM'12) received the Laurea degree in electronic engineering and the $\mathrm{Ph} . \mathrm{D}$. degree in electronics and automation engineering from the University of Catania, Italy, in 1999 and 2003, respectively.

Since 1999 he has been with the Radio Frequency Advanced Design Center (RF-ADC), a joint research center supported by the University of Catania and STMicroelectronics. In 2003 and 2008, he joined the Faculty of Engineering at the University of Catania as Research Associate and Research Assistant Professor, respectively. Since October 2011, he has been with STMicroelectronics, Catania, Italy. He was involved in the design and development of silicon-integrated transceivers for wireless communications. His research interests also included mm-wave integrated circuits for communications, radar and medical applications. He is co-author of more than 50 papers in international journals and conference proceedings, several patents, and a book on integrated inductors and transformers for RF/mm-wave applications. Over the last few years, he has been also active in the field of organic electronics within the framework of European Research Projects.

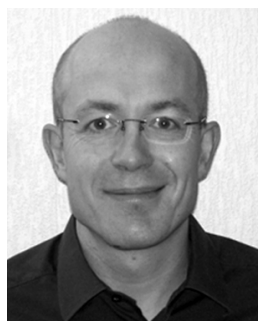

Romain Gwoziecki received the Ph.D. degree in microelectronics in 1999 in the field of deep submicron CMOS transistors architectures.

From 2000 to 2001, he was with STMicroelectronics, Agrate, Italy, participating to the integration of embedded Flash. From 2001 to 2007, he was with CEA-LETI, in the framework of the Crolles2-Alliance, Grenoble (France) working on advanced CMOS technologies (from $130 \mathrm{~nm}$ down to $65 \mathrm{~nm}$ ) on Bulk and SOI substrates. Since 2007, he has been working on the development of printed organic electronics, with special focus on characterization and modeling.

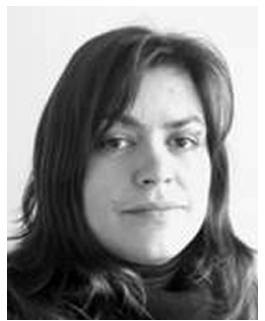

Stephanie Jacob received the M.S. degree in microelectronics and telecommunications from the "Ecole Polytechnique Universitaire de Marseille," France, in 2004 and the Ph.D. degree in microelectronics from the University of Provence, France, in 2008. Her Ph.D. work was carried out in CEA-LETI and ATMEL Rousset, France, on the integration, characterization and modelling of silicon nanocrystal memories.

Since 2008, she has joined CEA-LITEN, France to work on the electrical characterization and modelling of organic transistors on flexible substrate. She is the co-author of more than 20 papers in international journals and conference proceedings.

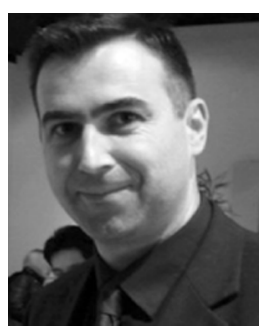

Nenad Marjanović received the MSc. degree in materials science at the Faculty of Electrical Engineering, University of Belgrade, Serbia.

After that, he joined the group of Prof. Niyazi Serdar Sariciftci at Johannes Kepler University Linz, Austria, where he received the PhD. Degree in Technical Sciences. The device physics and photo-induced effects in organic thin film transistors and diodes were in the focus of his scientific activity. At the end of 2005 he co-founded the company plastic electronic $\mathrm{GmbH}$ in Linz, Austria, where he held a CTO position. During that period he and his team developed the technology for the fabrication of organic electronic devices (e.g., OTFTs and diodes) in a roll-to-roll manufacturing fashion combining printing and vacuum evaporation. In 2009 he joined the group of Prof. Reinhard R. Baumann at Chemnitz University of Technology and Fraunhofer Institute for Electronic Nano Systems ENAS in Chemnitz, Germany, where he worked on developing the technology for low temperature curing of printed amorphous metal oxides on the substrates commonly used in Printed Circuit Board (PCB) industry. In 2011 he joined CSEM Muttenz, Switzerland, where he is currently taking a
Project Manager position. He focuses on the development of hybrid printed electronics - coupling printed and silicon based electronic components on flexible PCB. He has published more than 17 peer-reviewed papers in high level scientific journals, which are cited more than 680 times.

Dr Marjanović was awarded with the Serbian National Prize "Nikola Tesla" for Science and Technological Innovations by the Serbian Government in 2010.

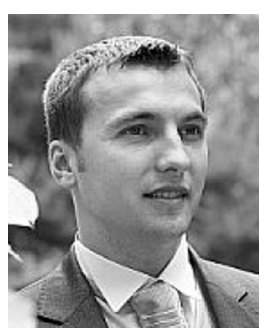

Marek Chrapa received the MSc. degree in materials engineering from Wroclaw University of Technology, Poland (2006). His master thesis was about kinetics of photochromic reaction of azobenzene derivatives of poly-methyl-methacrylate (polymers for optical data storage) and was done at Institute of Physical and Theoretical Chemistry where he worked under supervision of the Dr. K. Matczyszyn and Prof. J. Sworakowski.

In 2006 he moved to Switzerland to join CSEM (Swiss Center for Electronics and Microtechnology). Since then he is R\&D and process engineer responsible for process development and project support in the domain of organic and printed electronics. His main research and engineering activities are focused on the development of OLED, OPV, OPD, OTFT, sensors, functionalized coatings and encapsulation. Currently he is developing a process for fabrication of all printed organic tandem solar modules. The demonstrator of this device was shown at LOPEC conference in Munich (June 2013).

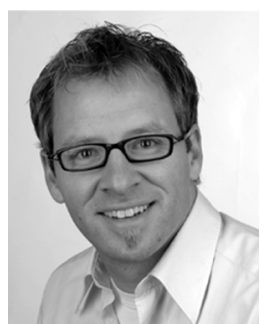

Jürg Schleuniger received a diploma as Senior Scientific Laboratory Technician HFP and is currently doing an extra-occupational study BSc in engineering management.

In the last few years, he has gained professional experience in the field of printed electronics and LCD display technologies. In 2003 he joined EMPA (Swiss Federal Laboratory for Material Science and Testing) in the Laboratory for Functional Polymers, where he worked in research of organic solar cells. In addition to the synthesis of semi conductive materials as electron donor, he was involved in the developing of processes to fabricate solar cell. In 2006, he moved on to the research group of ROLIC Technologies, Allschwil, Switzerland, where he worked in research and development in the field of liquid crystal polymers for displays and optical films. Furthermore he made a feasibility study to test the potential of ROLIC materials for organic photovoltaic. Since 2008 he is R\&D engineer at CSEM in the field of printed electronics which includes process development for fabricating OLEDs, OPV and OTFTs.

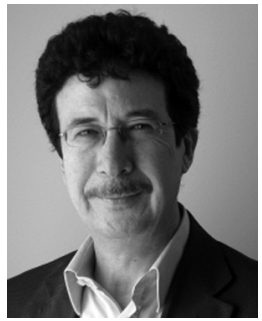

Giuseppe Palmisano (M'08-SM'09) received the Laurea degree in electronic engineering from the University of Pavia, Pavia, Italy, in 1982.

From 1983 to 1991 , he was a Researcher with the Department of Electronics, University of Pavia, where he was involved in CMOS and BiCMOS analog integrated circuit design. In 1992, he was a Visiting Professor with Universidad Autonoma Metropolitana (UAM), Mexico City, Mexico, where he held a course on microelectronics for Ph.D. students. In 1993 and 2000, he joined the Faculty of Engineering at the University of Catania, Catania, Italy, as an Associate Professor and Full Professor, respectively, teaching microelectronics. Since 1999, he has been leading the Radio Frequency Advanced Design Center (RF-ADC), a joint research center supported by University of Catania and STMicroelectronics, Catania, Italy. He has supervised the design of several innovative analog integrated circuits and systems within the framework of national and European research projects and in collaboration with electronic industries. He is the coauthor of more than 250 papers in international journals and conference proceedings, two books, and several international patents. His current research interest lies in the design of RF and microwave integrated circuits for wireless communications. 